\title{
Fuzzy-Based Electric Current Dependency on an MR Damper Model
}

\author{
Javier A. Ruiz-Cabrera, Ruben Morales-Menendez, \\ Luis E. Garza-Castañon, Ricardo A. Ramirez-Mendoza, and Olivier Sename.
}

\begin{abstract}
A Magneto-Rheological (MR) damper exhibits a hysteretic and non-linear behavior. This behavior makes it a challenge to develop a model for the system. The present research is centered on proposing and analyzing a fuzzy-based method employed to introduce the electric current dependency into $M R$ damper models, based on experimental data. Among the state of the art, a semi-phenomenological model was selected. The fuzzy-based method was compared against other approaches. The results showed that the fuzzy-based method was able to correctly introduce the dependency of the model to the electric current and reduce the prediction error by more than $12 \%$.
\end{abstract}

\section{MOTIVATION}

With the development of science and technology for automobiles and the continuously increasing need for safety and comfort, great attention has been drawn to automotive suspension systems. Semi-active suspension systems have been specially analyzed. These systems offer the reliability of passive devices, but maintain the versatility and adaptability of active systems. A semi-active suspension can be adjusted in real time, but cannot input energy into the system being controlled. Hence, the force delivered by the suspension is constrained to be proportional and opposite to the elongation speed of the damper. Nonetheless, the power requirement of these systems is considerably lower than that of an active system.

A widely investigated mono-tube semi-active damper is the one denominated Magneto-Rheological $(M R)$ damper. An $M R$ damper is a non-linear dynamical system where the inputs can be the elongation speed and an electric current. The electric current is the control input that modulates the damping characteristic of the $M R$ fluid through the variation of a magnetic field.

$M R$ fluids are unique due to their ability to change their properties reversibly between fluid and solid-like states upon the application of a magnetic field. As discussed in [1], when a certain magnetic field is applied to an $M R$ fluid, the particles in the fluid become polarized and form polarization chains in the direction parallel to the applied field. Fig. 1 shows the force-velocity behavior of an industrial $M R$ damper under various constant electric current inputs.

J. A. Ruiz-Cabrera is an MSc student at Tec de Monterrey, Monterrey, NL, Mexico. javier.ruiz.cabreraditesm.mx

R. Morales-Menendez, L. E. Garza-Castañon, and R. A. RamirezMendoza are with Tec de Monterrey, Monterrey, NL, Mexico. \{rmm, legarza, ricardo.ramirez\}@itesm.mx

O. Sename is with INPG, olivier.senamedgipsa-lab.inpg.fr

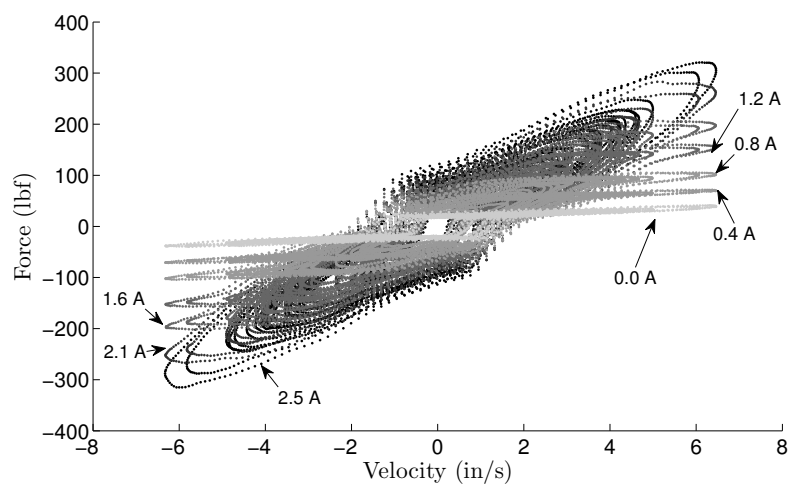

Fig. 1. Force-velocity behavior of an industrial $M R$ damper. The force is plotted against the velocity for various constant electric current inputs.

According to [2], concerning the automotive industry $M R$ fluids are appealing for vehicle suspension systems since they can operate at temperatures ranging from 40 to $150{ }^{\circ} \mathrm{C}$ with only slight variations in the yield stress. Additionally, $M R$ fluids are almost insensitive to impurities and can be controlled with low voltages and an electric current driven power supply outputting 1-2 $A$.

Although $M R$ dampers are greatly promising for the control of vehicle suspension systems, their major drawback lies on their non-linear and hysteretic behavior. Furthermore, the first step in designing a control strategy for a suspension system is, usually, modelling the behavior of the damper in an accurate manner. Different modelling techniques have been studied for $M R$ dampers. In [1], [3], and [4] phenomenological modelling techniques were explored. In [5], semi-phenomenological techniques were used to develop a mathematical model able to describe the hysteretic behavior of an $M R$ damper. In [2] and [6] black-box models based on Non-linear Autoregressive with eXogenous terms (NARX) structures were studied.

The present study is motivated on the aforementioned challenge that involves the correct modelling of an $M R$ damping system, with specific concern on the inclusion of the electric current dependency to the models. Among the state of the art, the semi-phenomenological model presented in [5] was selected for analysis. This analysis is meant to compare a polynomial method for including the electric current on the model, versus a fuzzy-based technique. Experimental data sets were obtained at an industrial testing laboratory. Section II presents a literature review. Section III discusses the experimental setup and describes the design of experiments. 
TABLE I

DESCRIPTION OF VARIABLES

\begin{tabular}{|c|c|}
\hline Variable & Description \\
\hline \hline$F(t)$ & $M R$ damper force \\
$F_{\text {est }}(t)$ & Estimated $M R$ damper force \\
$I(t), i(t)$ & Electric current \\
$x(t)$ & Damper piston position \\
$\dot{x}(t), v(t)$ & Damper piston velocity \\
$A_{1}$ & Dynamic yield force of the $M R$ fluid \\
$A_{2}$ & Post-yield viscous damping coefficient \\
$A_{3}$ & Pre-yield viscous damping coefficient \\
$V_{0}$ & Hysteretic critical velocity \\
$X_{0}$ & Hysteretic critical displacement \\
$d_{k j}$ & General coefficients for the $S-P$ model \\
\hline
\end{tabular}

Section IV presents the results. Section V discusses the obtained results. Finally, section VI concludes the research. Table I defines the variables that will be used through the paper.

\section{LITERATURE REVIEW}

\section{A. Previous Work}

The research done in [2] compared the semi-physical modified Bouc-Wen model presented in [4] and a black-box $N A R X$ model structure. In order to include the electric current dynamics into the models, two methods were employed. For the semi-physical model, each coefficient was made equal to a time varying linear function of the electric current. For the $N A R X$ model, two regressors (present and past values) for the input electric current were added. The reported results showed that the semi-physical model was not able to predict the behavior of the damper. On the other hand, the NARX model was observed to accurately predict the damping force of the system. In the research presented in [1], a phenomenological model based on the phase shifting dynamics of $M R$ fluids was discussed. The authors commented that in the model, all the coefficients are to be assumed dependent on the applied electric current. That is, the coefficients should be functions of the applied magnetic field. This dependency was said to be correctly approximated by a polynomial of order two.

\section{B. Semi-Phenomenological ( $S-P)$ Model}

Among the state of the art, the semi-phenomenological model for $M R$ dampers presented in [5] has been greatly analyzed in the past years. The proposed model is said to describe the bi-viscous and hysteretic behaviors of the $M R$ damper with high precision. The structure is described in (1).

$$
\begin{array}{r}
F(t)=A_{1} \tanh \left(A_{3}\left(\dot{x}(t)+\frac{V_{0}}{X_{0}} x(t)\right)\right)+ \\
A_{2}\left(\dot{x}(t)+\frac{V_{0}}{X_{0}} x(t)\right)
\end{array}
$$

In the equation, the model can be seen to use the displacement and velocity as inputs and only depend on five parameters. The authors used a non-linear least-squares algorithm in order to identify the coefficients of the model. The results obtained in the experimentation were said to prove the correctness of the proposed structure. In addition, the concise form of the model was mentioned as its best feature. Nevertheless, the authors did not use experiments in which the electric current varied over time. Although, it was noted that each parameter could be made equal to a function of the electric current in order to include varying electric current scenarios into the model.

\section{Fuzzy-Based Systems}

Fuzzy systems have been recently employed for modelling and control of physical processes. A fuzzy system is a static nonlinear mapping between inputs and outputs. Fig. 2 presents a block diagram of a general fuzzy system. The inputs and outputs of the system are crisp, that is, they are real numbers and not fuzzy sets. The fuzzyfication block converts the crisp inputs to fuzzy sets (membership functions), the inference mechanism uses the fuzzy rules in the rule-base to produce fuzzy conclusions, and the defuzzification block converts these fuzzy conclusions into crisp outputs.

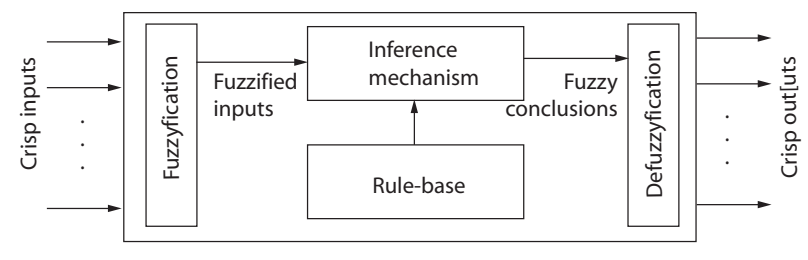

Fig. 2. Fuzzy system block diagram.

Among fuzzy-based systems, a Takagi-Sugeno-Kang (TSK) fuzzy system is one whose output conclusions are linear functions. These fuzzy systems have been greatly utilized to model the behavior of $M R$ dampers. In [7] and [8], $T S K$ models of the $M R$ damper were presented. The selected fuzzy structures utilized three inputs (displacement, velocity, and voltage) and one output (damping force). The total number of fuzzy rules was 27 per structure and the output functions were linear. A similar approach was followed in [9]. In the work done in [10], a self-tuning fuzzy structure was analyzed to model an $M R$ damper. As inputs for the model were selected the displacement, velocity, and electric current, each with five triangular membership functions. The output functions were selected as constants combined using the centroid method. Finally in [11], direct and inverse fuzzybased models of the $M R$ damper were presented. The fuzzy structure for the direct model employed velocity, acceleration, and control voltage as inputs and damping force as output. On the other hand, for the inverse model the control voltage and damping force were swapped with respect to the direct one.

\section{DESIGN OF EXPERIMENTS}

\section{A. Experimental System}

The implemented experimental system can be divided into four parts: an $M R$ damper, the actuators, the control system, and the data acquisition system. An $M R$ damper, 
part of a suspension system from a commercial automobile, was employed. A controller testing system was used to control the position of the damper. A data acquisition system commanded the controller and recorded the position and force of the $M R$ damper, as well as the electric current on the coil. A sampling frequency of $512 \mathrm{~Hz}$ was used. The displacement actuator was a hydraulic servo-controlled piston of 3000 psi and displacement bandwidth of $15 \mathrm{~Hz}$. The displacement and electric current ranges were: 0 - 1.6 in, and $0-2.5 A$, respectively. The damping force was measured using a load cell and the measured span was 0 - $640 \mathrm{lbf}$. The experimental setup was controlled and monitored by a Human-Machine Interface (HMI) developed in a commercially available software.

\section{B. Design of Experiments}

Experiments were designed in order to generate displacement and electric current input patterns that would characterize the behavior of an $M R$ damper for automotive applications. Special attention was placed on the proper frequency content of the displacement signals. Additionally, the sequences were selected in order to aid the modelling process of the system. The experiments were based on the work presented in [12], where a set of training sequences was reviewed and designed for the identification of $M R$ dampers.

Eight sets of experimental data were obtained for the identification of $M R$ damper models. In the selected experiments, the electric current sequences were: Constant Steps (CS), Increased Clock Period Signal (ICPS), Pseudo-Random Binary Sequence (PRBS), and Amplitude Pseudo-Random Binary Sequence (APRBS). On the other hand, Road Profile $(R P)$ sequences were employed as displacement inputs. These $R P$ sequences were calculated for smooth highways, and were based on the work in [13] and [14]. Three $30 \mathrm{~s}$ experiments were performed for the highly varying electric current sequences. In addition, two $600 s$ experiments with $A P R B S$ and ICPS electric current sequences were performed in order to test the behavior of the $M R$ damper as the temperature of the device increased. Finally, three $210 \mathrm{~s}$ experiments were carried out employing $C S$ electric current sequences. For these last experiments, the electric current was held constant at seven different values $(0,0.4,0.8,1.2$, 1.6, 2.1, $2.5 A$ ). Various replicates of the eight experiments were performed and used as validation data.

The specific sequences of the eight experiments are shown in Table II, where the experiments have been labeled according to the sequences employed. The table specifies the utilized input sequences, and the number of replicates performed. Moreover, Figs. 3 and 4 show 10 and 40 second windows of the sequences employed for the first and last experiments, respectively. In addition, 30 second windows present the frequency content of those experiments.

\section{MODELLING RESULTS}

The modelling results were compared by means of the Square Root of the Sum of the Squared Errors (RSSE) and the Error to Signal Ratio (ESR) indexes. The RSSE and
TABLE II

EXPERIMENTAL DATA SETS

\begin{tabular}{|l|c|c|c|}
\hline $\begin{array}{l}\text { Experiment } \\
\text { Name }\end{array}$ & $\begin{array}{c}\text { Displacement } \\
\text { Pattern }\end{array}$ & $\begin{array}{c}\text { E. Current } \\
\text { Pattern }\end{array}$ & $\begin{array}{c}\text { Number of } \\
\text { Replicates }\end{array}$ \\
\hline \hline RP-ICPS & RP & ICPS & 11 \\
RP-APRBS & RP & APRBS & 11 \\
RP-PRBS & RP & PRBS & 11 \\
RP-APRBS-L & Long RP & APRBS & 3 \\
RP-ICPS-L & Long RP & ICPS & 4 \\
RP1-CS & RP & CS & 1 \\
RP2-CS & RP & CS & 1 \\
RP3-CS & RP & CS & 1 \\
\hline
\end{tabular}

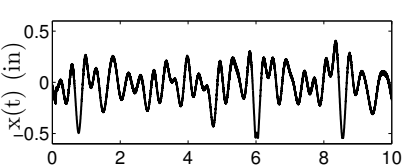

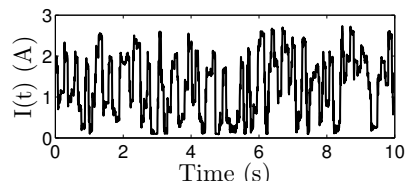

(a)

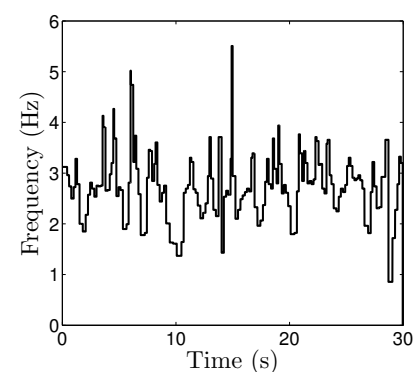

(b)
Fig. 3. Description of experiment RP-ICPS. Displacement and electric current patterns (left). Frequency content (right).

$E S R$ are presented in (2) and (3), respectively. The RSSE presents the square root of the sum of the errors between the predicted and experimental output forces normalized by the total number of samples. The ESR is the ratio of the sum of squared errors and the variance of the experimental force. This last index is equal to one if the model is trivial. Both indexes are equal to zero if the model is perfect.

$$
\begin{gathered}
R S S E=\sqrt{\frac{1}{T} \sum_{t=1}^{T}(F(t)-\hat{F}(t))^{2}} \\
E S R=\frac{(R S S E)^{2}}{\frac{1}{T} \sum_{t=1}^{T}\left(F(t)-\left(\frac{1}{T} \sum_{j=1}^{T} F(j)\right)\right)^{2}}
\end{gathered}
$$

The $S$ - $P$ model shown in (1) was identified for the first replicate of each of the eight sets of experiments afore-

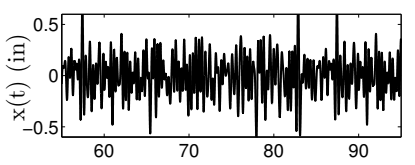

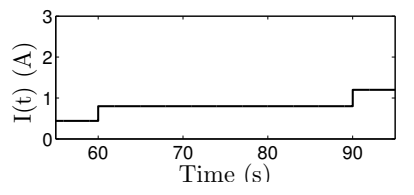

(a)

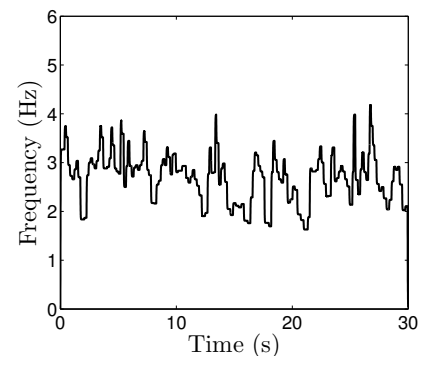

(b)
Fig. 4. Description of experiment RP3-CS. Displacement and electric current patterns (left). Frequency content (right). 
mentioned. The identification algorithm was chosen as nonlinear least squares. The five coefficients of the model were randomly initialized 25 times and the lowest error value was recorded. The resulting identification errors are shown in Table III.

TABLE III

IDENTIFICATION ERRORS FOR THE ORIGINAL $S$ - $P$ MODEL

\begin{tabular}{|c|c|c|}
\hline Model Training & RSSE $(l b f)$ & ESR \\
\hline \hline RP-ICPS & 38.35 & 0.1681 \\
RP-APRBS & 31.23 & 0.1098 \\
RP-PRBS & 44.74 & 0.3410 \\
RP-APRBS-L & 27.27 & 0.0837 \\
RP-ICPS-L & 35.95 & 0.1472 \\
RP1-CS & 43.18 & 0.2073 \\
RP2-CS & 45.16 & 0.2264 \\
RP3-CS & 44.79 & 0.2105 \\
\hline
\end{tabular}

It can be noticed that the $S$-P model obtained high identification errors for most of the experiments. A later cross validation was performed using all the data sets and replicates (a total of 43 experiments). This validation confirmed that the $S-P$ model was not able to predict the damping force in an accurate manner. Even for the models that obtained the lowest identification errors, ESR values of more than 0.30 were observed.

In order to include the electric current into the model, each of the parameters $\left(A_{1}, A_{2}, A_{3}, V_{0}\right.$, and $\left.X_{0}\right)$ was made equal to a second order polynomial dependent on the electric current. The new structure depended on 15 coefficients that were identified for the first replicate of each of the eight sets of experimental data.

Contrary to the original one, the electric current dependent $S-P$ model was able to obtain low error values for all the experimental data sets. A considerable decrease in error was seen for the models trained using experiments with constant electric current. As for the original model, a cross validation was performed using the electric current dependent version. Figs. 5 and 6 present box and whisker plots for the RSSE and ESR by trained model, respectively.

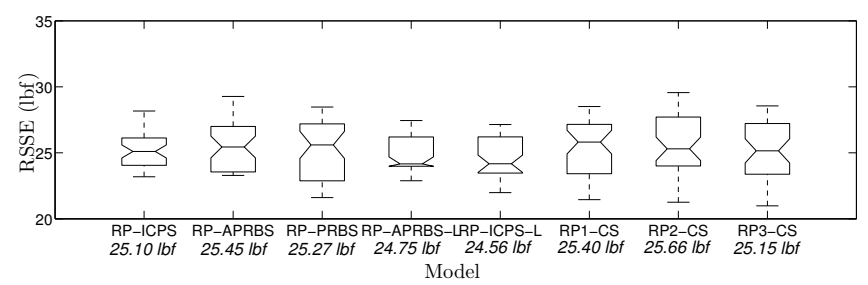

Fig. 5. RSSE results for the electric current dependent $S$ - $P$ model. The boxes have lines at the lower quartile, median, and upper quartile values. The whiskers are lines extending from each end of the boxes to show the extent of the rest of the data. Outliers are data with values beyond the ends of the whiskers. The average error is shown at the bottom of the plot.

Opposed to the use of polynomials in order to include the electric current to the $S-P$ model, a fuzzy-based approach is analyzed in the present work, as proposed in [15]. The fuzzy-based structure employs the electric current as input, and the fuzzy rules are defined as specified in (4).

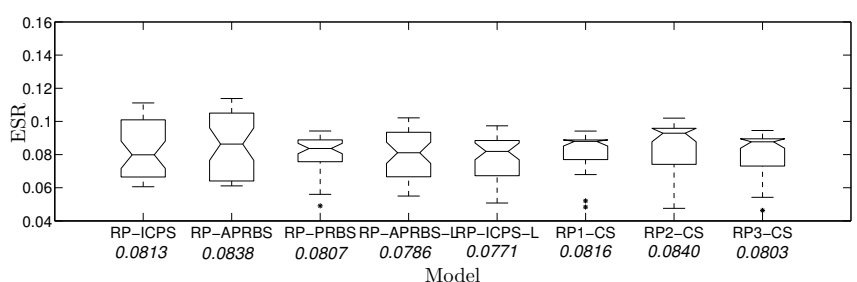

Fig. 6. ESR results for the electric current dependent $S-P$ model.

$$
\text { If } i(t) \text { is } M_{F j} \text { then } f_{j}(t)=g_{j}(x(t), \dot{x}(t))
$$

Notice that each output function $f_{j}(t)$ depends on the displacement and the velocity of the $M R$ damper. $M_{F j}$ are fuzzy sets of $i(t)$. The output functions for the model were selected to be of the form of the $S$ - $P$ model of the $M R$ damper presented in [16] and shown in a general form in (5).

$$
\begin{array}{r}
f_{j}(t)=d_{1 j} \tanh \left(d_{2 j}\left(\dot{x}(t)+d_{3 j} x(t)\right)\right) \\
+d_{4 j}\left(\dot{x}(t)+d_{3 j} x(t)\right)
\end{array}
$$

The overall output force of the damper was selected to be computed as specified by (6),

$$
F(t)=\frac{\sum_{j=1}^{7} W_{F j}(i(t)) f_{j}(t)}{\sum_{j=1}^{7} W_{F j}(i(t))}
$$

where $W_{j}$ represents the membership degree of $i(t)$ on each of the membership functions. Fig. 7 depicts the proposed fuzzy-based structure.

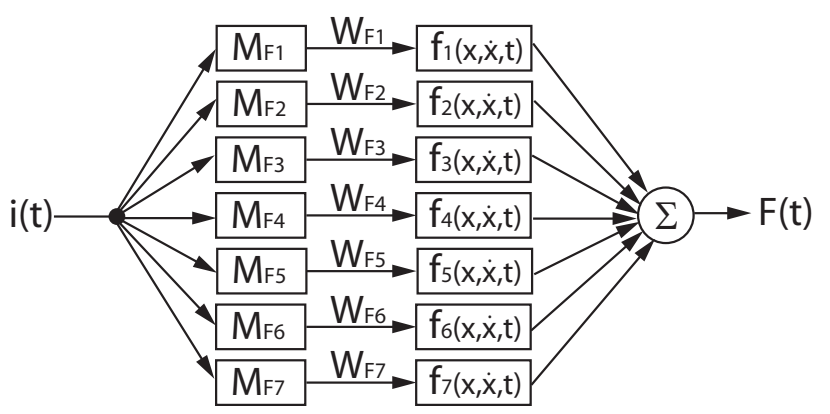

Fig. 7. Fuzzy-based electric-current-dependent structure.

As the original $S$ - $P$ model only depends on the displacement and velocity of the $M R$ damper, the experimental data sets with constant stepped increments of the electric current were selected as identification sets. Each of these experimental data sets $(R C 1-C S, R C 2-C S$, and $R C 3-C S)$ was broken into seven subsets, each corresponding to a time span with constant electric current values. Then, the coefficients in (5) were identified using non-linear least squares and yielded one $S$ - $P$ model for each of the seven electric current stepped increments on the experiments. In this manner, one fuzzy-based electric-current-dependent structure, with seven output functions, was obtained from experiment $R C l-C S$, one 
from experiment $R C 2-C S$, and one from experiment $R C 3$ $C S$. The fuzzy-based structures were labeled according to the experimental data set with which they were trained. The input membership functions for each structure were defined as seven Gaussian functions with variance equal to 0.2 and means of $0,0.4,0.8,1.2,1.6,2.1$, and $2.5 A$, respectively.

Once the three structures were trained, a cross validation was performed using the eight sets of experimental data with replicates. Figs. 8 and 9 present the resulting $R S S E$ and ESR by trained structure, respectively. Notice that the three fuzzy-based structures obtained validation errors with medians below $29 l b f$ and 0.1 .

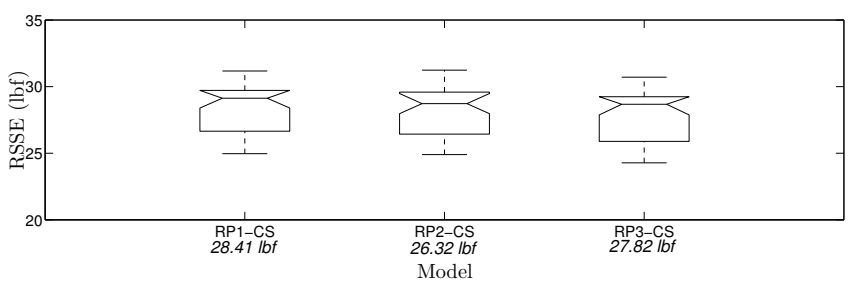

Fig. 8. RSSE results for the fuzzy-based electric-current-dependent $S$-P model.

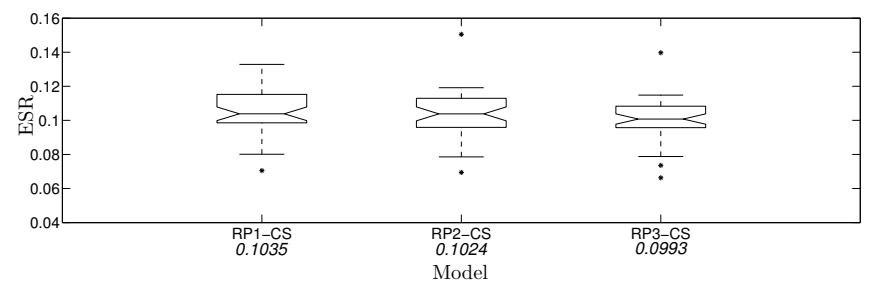

Fig. 9. $E S R$ results for the fuzzy-based electric-current-dependent $S$-P model.

\section{DISCUSSION}

\section{A. Best Models}

The average RSSE and ESR values were calculated for the original $S$ - $P$ model and its two electric-current-dependent variations. The lower average errors, and the corresponding experimental data sets with which the structures were trained, are shown in Table IV. Notice that the experimental data sets with which the lowest $R S S E$ values were obtained correspond to those of the ESR.

TABLE IV

BEST AVERAGE RSSE AND ESR B Y EXPERIMENTAL DATA SET.

\begin{tabular}{|l|c|c|c|}
\hline Model & Exp. Data Set & RSSE [lbf] & ESR \\
\hline \hline Original S-P & RP1-CS & 39.86 & 0.2161 \\
Polynomial Method S-P & RP-ICPS-L & 24.56 & 0.0771 \\
Fuzzy-Based Method S-P & RP3-CS & 27.82 & 0.0993 \\
\hline
\end{tabular}

The best structures corresponding to the two methods for including the electric current to the $S$ - $P$ model were further compared by means of force-time and force-velocity plots. Experiment $R P-A P R B S$ was employed to test the models in the time domain, due to its variant electric current content. Experiment RPI-CS was employed to test the models in the force-velocity behavior, due to its constant electric current increments.

\section{B. Polynomial Method}

The polynomial inclusion of the electric current to the $S-P$ model diminished the RSSE and ESR values by $15 \mathrm{lbf}$ and 0.14 , respectively for the best structure. Nonetheless, this inclusion of the electric current incremented considerably the number of parameters. Fig. 10 presents a two second window that compares the experimental force and the force estimated by the model. Fig. 11 compares the force-velocity behavior of the experimental and estimated damper forces at six different constant electric current values.

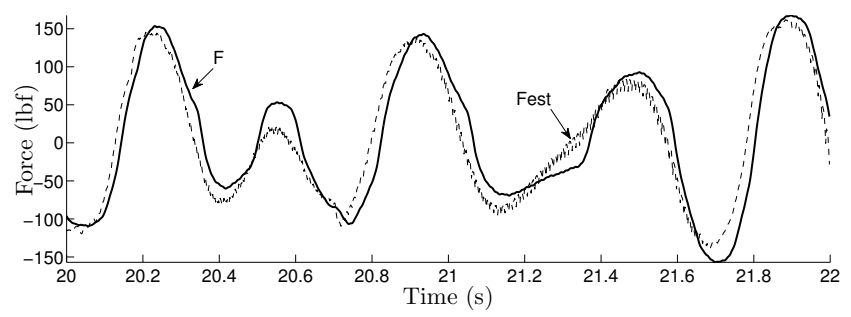

Fig. 10. Experimental and estimated damper force by the selected $S$ $P$ model with polynomial electric current dependency. Experimental data taken from experiment $R P-A P R B S$.

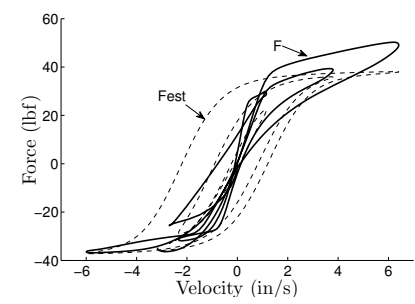

(a) $0.0 \mathrm{~A}$

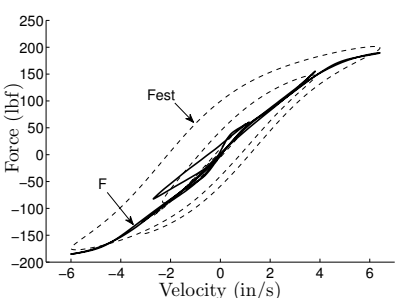

(c) $1.6 \mathrm{~A}$

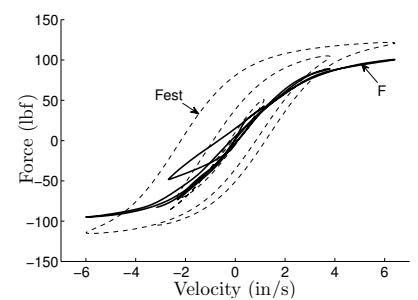

(b) $0.8 \mathrm{~A}$

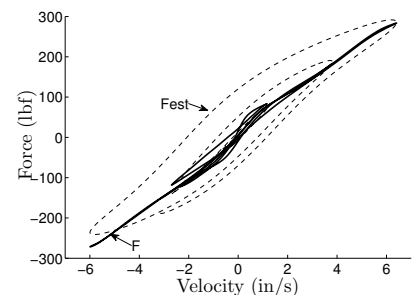

(d) $2.5 \mathrm{~A}$
Fig. 11. Experimental and estimated F-v behavior of the selected $S$ - $P$ model with polynomial electric current dependency. Each of the figures presents the behavior at a constant value of the electric current using the data from experiment $R P 1-C S$.

It can be seen from the force-time plot that the polynomial electric-current-dependent $S-P$ model follows the pattern of the experimental force with a minor lead time. On the other hand, the force-velocity plots confirm that the $S-P$ model correctly follows the non-linearities of the $M R$ damper, but overly exaggerates the width of the hysteresis loop. The model seemed to improve its performance as the electric current was increased. 


\section{Fuzzy-Based Method}

The proposed non-linear fuzzy-based model was observed to reduce the error indexes by $12 \mathrm{lbf}$ and 0.12 with the best structure. A force-time comparison revealed that the proposed fuzzy-based structure acceptably followed the experimental force. Nonetheless, minor noise was observed at certain moments, which may be produced by the shifting dynamics of membership functions. Moreover, Fig. 12 compares the force-velocity behavior of the experimental and estimated damper forces at six different constant electric current values.

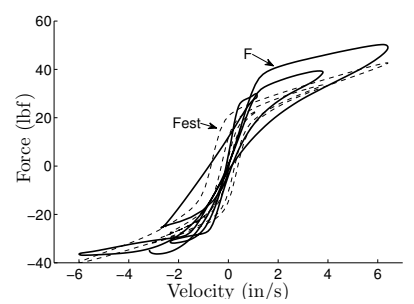

(a) $0.0 \mathrm{~A}$

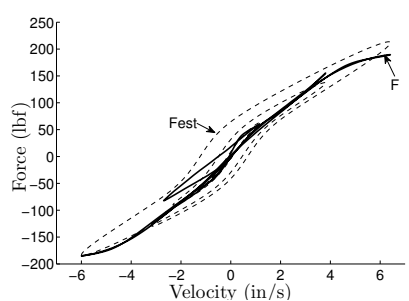

(c) $1.6 \mathrm{~A}$

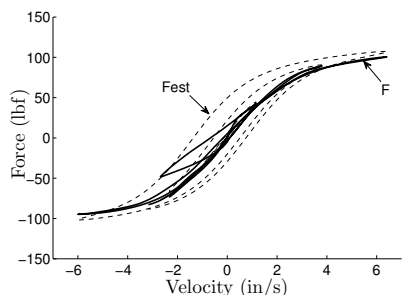

(b) $0.8 \mathrm{~A}$

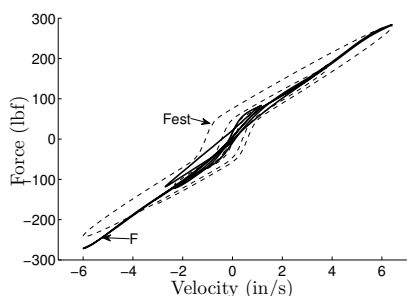

(d) $2.5 \mathrm{~A}$
Fig. 12. Experimental and estimated F-v behavior of the selected $S-P$ model with fuzzy-based electric current dependency. Each of the figures presents the behavior at a constant value of the electric current using the data from experiment $R P 1-C S$.

The force-velocity plots confirm that the $S$ - $P$ model with fuzzy-based electric current dependency accurately follows the non-linear behavior of the $M R$ damper. Nevertheless, the hysteresis loops can be observed to be slightly wide in comparison to the experimental force. Moreover, as the electric current increases the proposed structure was seen to improve its performance.

\section{CONCLUSIONS}

The electric-current-dependent $S$ - $P$ model obtained low error indexes when employing the polynomial method with 10 parameters. Nevertheless, as it was observed in the forcevelocity plots, the model could not accurately predict the non-linear and hysteretic behavior of the $M R$ damper. This performance may be in part due to the restrictive way in which the electric current dependency was introduced to the model.

On the other hand, the proposed fuzzy-based approach to introduce the electric current dependency was observed to obtain RSSE and ESR values slightly higher than those obtained with the polynomial method. Nonetheless, the forcevelocity plots allowed to see that the fuzzy-based structure closely mimicked the non-linear and hysteretic behavior of the $M R$ damper. After the present analysis, the fuzzybased method can be regarded as a considerable option for including the electric current dynamics into an $M R$ damper $S$ - $P$ model. Furthermore, if more closely spaced steps of the electric current were performed during the experimentation phase, the performance of the proposed fuzzy-based structure may see a significant improvement. Additionally, other $M R$ damper models could be employed as output functions for the fuzzy structure.

\section{ACKNOWLEDGMENTS}

The authors thank the Autotronics and Supervision and Advanced Control research chairs at Tec de Monterrey for their partial support.

\section{REFERENCES}

[1] L. X. Wang and H. Kamath. Modelling Hysteretic Behaviour in MR Fluids and Dampers using Phase-Transition theory. Smart Mater. Struct., 15:1725-1733, 2006.

[2] S. M. Savaresi, S. Bittanti, and M. Montiglio. Identification of Semi-Physical and Black-Box Non-Linear Models: the Case of MRDampers for Vehicles Control. Automatica, 41(1):113-127, 12005.

[3] N. M. Kwok, Q. P. Ha, T. H. Nguyen, J. Li, and B. Samali. A Novel Hysteretic Model for Magnetorheological Fluid Dampers and Parameter Identification using Particle Swarm Optimization. Sensors and Actuators A: Physical, 132(2):441-451, 11/20 2006.

[4] B. F. Spencer, S. J. Dyke, M. K. Sain, and J. D. Carlson. Phenomenological Model of a MR Damper. ASCE J. of Engineering Mechanics, 1996.

[5] S. Guo, S. Yang, and C. Pan. Dynamical Modeling of MagnetoRheological Damper Behaviors. Intelligent Material Systems and Structures, 17:3-14, 2006.

[6] E. Niño-Juarez, R Morales-Menendez, R Ramirez-Mendoza, and L Dugard. Minimizing the Frecuency in a Black Box Model of a Magneto-Rheological Damper. In $11^{\text {th }}$ Mini Conf. on Vehicle Sys. Dyn., Ident. and Anomalies, 2008.

[7] K. C. Schurter and P. N. Roschke. Fuzzy Modeling of a Magnerorheological Damper using ANFIS. In Fuzzy Systems, 2000. FUZZ IEEE 2000. The $9^{t h}$ IEEE Int. Conf. on, volume 1, pages 122-127 vol.1, 2000.

[8] V. S. Atray and P. N. Roschke. Design, Fabrication, Testing, and Fuzzy Modeling of a Large Magnetorheological Damper for Vibration Control in a Railcar. In Proc. of the 2003 IEEE/ASME J. Rail Conf., pages $223-229,2003$.

[9] H. Du and N. Zhang. Evolutionary Takagi-Sugeno Fuzzy Modelling for MR Damper. In $6^{\text {th }}$ Int. Conf. on Hybrid Intelligent Systems, pages $69-69$, Dec. 2006.

[10] K. K. Ahn, M.A. Islam, and D.Q. Truong. Hysteresis Modeling of Magneto-Rheological (MR) Fluid Damper by Self Tuning Fuzzy Control. In Int. Conf. on Control, Automation and Systems, pages $2628-2633$, oct. 2008.

[11] H. Wang and H. Hu. The Neuro-fuzzy Identification of MR Damper. In Fuzzy Systems and Knowledge Discovery, 2009. FSKD '09. $6^{\text {th }}$ Int. Conf. on, volume 6, pages $464-468$, aug. 2009.

[12] J. Lozoya-Santos, R. Morales-Menendez, and R. A. RamirezMendoza. Design of Experiments for MR Damper Modelling. In $17^{\text {th }}$ Int. J. Conf. on Neural Networks, pages 1915-1922, 2009.

[13] J. Y. Wong. Theory of Ground Vehicles. John Wiley, 2001.

[14] J. G. S. da Silva. Dynamical Performance of Highway Bridge Decks with Irregular Pavement Surface. Computers and Structures, 82:871$881,2004$.

[15] J. Ruiz-Cabrera and R. Morales-Menendez. MR Damper Fuzzy-Based Modelling. In $7^{\text {th }}$ EUROSIM Congress on Modelling and Simulation, 2010.

[16] S. Guo, S. Li, and S. Yang. Semi-active Vehicle Suspension Systems with Magnetorheological Dampers. In IEEE Int. Conf. on Vehicular Electronics and Safety, pages 403-406, 2006. 